ISSN: 1641-4713; e-ISSN: 2081-1160

DOI: https://doi.org/10.36551/2081-1160.2020.25.103-122

\title{
Las huellas de la contradicción entre desarrollo y ambiente. Un análisis del metabolismo socioeconómico en América del Sur a través de sus huellas ecológica e hídrica
}

Footprints of the Contradiction Between Development and Environment: An Analysis of Socioeconomic Metabolism in South America Through Its Ecological and Water Footprints

Guillermo Peinado

Instituto de Investigaciones Económicas Facultad de Ciencias Económicas y Estadística

Universidad Nacional de Rosario, Argentina Asociación Argentino-Uruguaya de Economía Ecológica ID ORCID: https://orcid.org/0000-0003-1100-4296 E-mail: gpeinado@fcecon.unr.edu.ar

Ain Mora

Instituto de Investigaciones Económicas Facultad de Ciencias Económicas y Estadística

Universidad Nacional de Rosario, Argentina Asociación Argentino-Uruguaya de Economía Ecológica

ID ORCID: https://orcid.org/0000-0002-8694-121X E-mail: amora@fcecon.unr.edu.ar Javier Ganem

Instituto de Investigaciones Económicas,

Facultad de Ciencias Económicas y Estadística

Universidad Nacional de Rosario, Argentina

ID ORCID: https://orcid.org/0000-0001-9559-8532

E-mail: jganem@fcecon.unr.edu.ar

Bruno Ferrari

Instituto de Investigaciones Económicas

Facultad de Ciencias Económicas y Estadística

Universidad Nacional de Rosario, Argentina

ID ORCID: https://orcid.org/0000-0002-6269-7291

bruno.ferrari@fcecon.unr.edu.ar

Recepción: 31.01 .2020

Aprobación: 12.03.2020

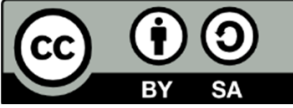


Resumen: A lo largo de la última mitad del siglo XX y a principios del siglo XXI se ha ido reconfigurando de diversas maneras la relación entre desarrollo y ambiente. A nivel general, actualmente, hay dos corrientes antagónicas en pugna: la que promueve el desarrollo sostenible, y la que a partir del oxímoron desarrollo y ambiente busca alternativas al desarrollo. Este trabajo busca aplicar indicadores para entender la estructura biofísica de América del Sur y brindar metodologías para nuevas alternativas al desarrollo. Para ello se desarrollan los indicadores biofísicos de huella ecológica y de huella hídrica, construidos en el marco de análisis de flujos de materiales del campo de la Economía Ecológica, que permitirán determinar la magnitud y las características de los flujos de materiales y energía que se derivan del perfil productivo y el patrón de inserción externo de América del Sur.

Palabras clave: economía política; economía ecológica; alternativas al desarrollo; desarrollo sustentable; desarrollo-subdesarrollo.

\begin{abstract}
Throughout the last half of the twentieth century and at the beginning of the twenty-first century, the relationship between development and environment has been reconfigured in various ways. At the general level there are currently two antagonistic currents in conflict: the one that promotes sustainable development, and the one that, from the oxymoron development and environment, seeks alternatives to development. This work seeks to apply biophysical indicators to understand the biophysical structure of South America and provide methodologies for new alternatives to development. For this, the biophysical indicators of ecological footprint and water footprint are developed, built within the framework of analysis of material flows of the field of Ecological Economics, which will allow to determine the magnitude and characteristics of the flows of materials and energy that are derived of the productive profile and the pattern of external insertion of South America.
\end{abstract}

Keywords: economic policy, ecological economics, alternatives to development, sustainable development, development-underdevelopment.

\title{
1. INTRODUCCIÓN
}

A lo largo de la última mitad del siglo XX y a principios del siglo XXI se ha ido reconfigurando de diversas maneras la relación entre desarrollo y ambiente. Si bien el devenir de esos debates excede a este trabajo ${ }^{1}$, se puede decir que, a nivel general, actualmente, hay dos corrientes antagónicas en pugna. La primera, hegemónica dentro de los diagramas de políticas públicas, se sintetiza en el concepto de desarrollo sostenible y promueve distintos objetivos políticos que buscarían un desarrollo alternativo a los viejos modelos desarrollistas ya que incorpora la noción socio-ambiental; es decir, la sustentabilidad de futuras generaciones en consonancia con un crecimiento económico sostenido en el tiempo. La segunda, un poco más actual, proviene de la ecología política y establece que los términos desarrollo y ambiente son contradictorios -esto es, un oxímoron- ya

\footnotetext{
${ }^{1}$ Para este debate ver, entre otros, Escobar (2014) y Estenssoro Saavedra y Vásquez Bustamante (2018).
} 
que el crecimiento económico -condición necesaria para el desarrollo- implica una mayor extracción de materiales y energía y, por ende, hay que buscar alternativas al desarrollo estableciendo nuevos parámetros para pensar nuevas alternativas post-desarrollistas.

La noción de desarrollo sostenible -o sustentable- fue expuesta en "Nuestro Futuro Común" (1987) -conocido como Informe Brundtland-, publicado por la Comisión Mundial sobre Medio Ambiente y Desarrollo a solicitud del Secretario General de las Naciones Unidas. La definición que se instaló y recorrió rápidamente por los discursos políticos de la época fue el entendimiento de que el desarrollo sostenible representaba el proceso que permite satisfacer las necesidades de la población actual sin comprometer la capacidad de satisfacer las necesidades de las generaciones futuras.

Bajo este paraguas teórico, y con las medidas económicas neoliberales implementadas en la década de los noventa en América del Sur, la dicotomía entre desarrollo y ambiente quedaba reducida a una cuestión de mecanismos de mercado en donde la resolución de los problemas de asignación de recursos se muestra eficiente para hacer coincidir las condiciones ecológicas y los valores culturales en un proceso económico en crecimiento. En esta mirada, la crisis ambiental no es un problema intrínseco del desarrollo sino un problema de mala asignación de los derechos de propiedad o de fallas de mercados, o incluso de fallas del Estado.

Esta racionalidad económica, que conlleva a la reconciliación entre ambiente y desarrollo, otorga un nuevo concepto de medio ambiente, en donde este se transforma en un recurso disponible que debe ser monetizado para asignarlo eficientemente mediante el sistema de precios o mediante el derecho de propiedad. La idea de desarrollo sostenible se presenta como un desarrollo alternativo que reconcilia epistemológica y políticamente la economía y la ecología.

Contrario a este proceso de globalización regido por la racionalidad económica y las leyes del mercado que predominaban en el discurso del desarrollo sostenible, emerge una nueva idea de sustentabilidad que se enraíza en bases ecológicas, en identidades culturales y en territorios de vida. El ecologismo popular -concepto desarrollado por el español Joan Martínez Alier-, se define como la expresión de una economía que se enfrenta a la mercantilización y se manifiesta en las fronteras extractivas (Martínez Alier, Sejenovich, Baud, 2015).

Aquí se aborda la interrelación entre economía y naturaleza de una forma diferente, entendiendo la economía como un subsistema abierto en constante interacción con los sistemas físico y social. Más allá de los valores monetarios de mercado que se aplicaba con el desarrollo sostenible, bajo este enfoque se 
pretende también la evaluación biofísica de los impactos ambientales de la economía humana, teniendo en cuenta indicadores biofísicos sobre flujos de energía o ciclos de materias primas, entre otros. De esta forma, se tienen en cuenta aspectos que tradicionalmente se dejaban de lado como son la disponibilidad de recursos y energía, y la generación de residuos.

Bajo estas premisas, este trabajo busca aplicar indicadores para entender la estructura biofísica de América del Sur y brindar metodologías para nuevas alternativas al desarrollo. La inserción en el mundo de América del Sur en los últimos años a partir de la acentuación de la matriz exportadora-primaria con eje en la extracción y exportación de recursos naturales y manufacturas derivadas, ha tenido consecuencias. Así, la explotación de la naturaleza ha asumido un lugar central en el patrón de acumulación y reproducción del capital. Ello ha reintroducido una serie de interrogantes vinculados a las posibilidades y a la sustentabilidad del modo de desarrollo vigente en los países de la región en el marco de su articulación con las necesidades de la acumulación global del capital (Belloni, Peinado, 2013) ${ }^{2}$.

En línea con estas problemáticas, en el siguiente apartado se introduce la noción de metabolismo socioeconómico, concepto que permite visibilizar los impactos ambientales que se derivan del perfil productivo y la inserción internacional extractiva de América del $\mathrm{Sur}^{3}$ en el período post-neoliberal (Belloni, Peinado, 2013). En el apartado tercero se desarrollan los indicadores biofísicos de huella ecológica y de huella hídrica. Dichos indicadores han sido construidos en el marco de análisis de flujos de materiales provenientes del campo de la Economía Ecológica. Estos permitirán en el cuarto apartado poner a prueba la hipótesis y determinar la magnitud de los flujos de materiales y energía que se derivan del perfil productivo y el patrón de inserción externo. Para ello se aplican los indicadores biofísicos de huella ecológica y de huella hídrica con el fin de visibilizar los impactos de las actividades económicas, y en especial del actual patrón de inserción externo, en el capital natural de las economías suramericanas. Por último, se presenta una serie de conclusiones que arrojan los indicadores y se aborda nuevamente la dicotomía entre desarrollo y ambiente.

\footnotetext{
${ }^{2}$ En relación a la sustentabilidad del modo de desarrollo, se entiende que la misma no puede condicionarse exclusivamente al ámbito productivo y distributivo, sino que debe de tener en cuenta el componente ambiental derivado de dichos procesos.

${ }^{3}$ Se han considerado las economías más grandes de la región: Argentina, Brasil, Bolivia, Chile, Colombia, Ecuador, Perú, Paraguay, Uruguay y Venezuela.
} 


\section{EL ESTUDIO DEL METABOLISMO SOCIOECONÓMICO}

El concepto de metabolismo socioeconómico -o metabolismo social, o metabolismo socioecológico - se ha extendido notablemente desde la década de 1990 a partir de los trabajos de Marina Fischer-Kowalski (1997; 1998), si bien permaneció de alguna manera latente en varios de los primeros exponentes de la Economía Ecológica ya en las décadas de 1960-1970 (Infante-Amate, González de Molina, Toledo, 2017).

Su importancia como herramienta teórica y metodológica radica en que permite visibilizar y estudiar las interacciones entre la sociedad y el medio. Lo hace desde una perspectiva holística o integradora, analizando su comportamiento biofísico, además de brindar información en simultáneo sobre los intercambios económicos y los intercambios ecológicos, en diferentes períodos de tiempo y escalas, expresando sus dimensiones materiales o tangibles (Toledo, 2013).

El mismo parte de la idea de que en toda sociedad y en todo tiempo, existe un ingreso y una salida de materiales y energía dentro de la misma. Ambos se convierten en productos manufacturados, en servicios y, finalmente, en desechos, contaminación o energía disipada. Ello puede ser entendido como el funcionamiento de un organismo vivo que metaboliza diferentes elementos ${ }^{4}$.

Esta idea del desenvolvimiento económico y social y sus impactos sobre el sistema natural pone de relieve que, en definitiva, todas las sociedades y sus regímenes económicos son dependientes porque requieren un flujo constante de materiales y energía. Estos luego son transformados, degradados y devueltos de alguna forma al sistema natural y, por lo tanto, deberían ser sustentables en el tiempo. Es decir, la sustentabilidad no es meramente un problema moral, sino que es, incluso, un problema material.

Adicionalmente, el enfoque del metabolismo socioeconómico permite alejarse de la crematística de los flujos monetarios para concentrarse en los flujos de materiales y de energía que la economía como disciplina tiende a invisibilizar. De hecho, Daly afirma que "los economistas se han concentrado demasiado en el sistema circulatorio de la economía y han omitido estudiar su tracto digestivo" (Daly, 2013: 135).

\footnotetext{
4 “A escala individual los seres humanos extraen de la naturaleza cantidades suficientes de oxígeno, agua y biomasa por unidad de tiempo para sobrevivir como organismos, y excretan calor, agua, dióxido de carbono y substancias mineralizadas y orgánicas. A escala social, el conjunto de individuos articulados a través de relaciones o nexos de diferentes tipos se organizan para garantizar su subsistencia y reproducción y extraen también materia y energía de la naturaleza por medio de estructuras colectivas y artefactos, y excretan toda una gama de residuos o desechos" (Infante-Amate, González de Molina, Toledo, 2017: 131).
} 
Para ello, se utilizan una serie de indicadores biofísicos bajo el paraguas del análisis del flujo de materiales y energía, que ofrece indicadores de extracción, consumo y comercio. Dicho enfoque puede realizarse tanto desde el lado de la producción, trabajando solo con los flujos directos de materiales y de energía, o bien a través del punto de vista del consumo, teniendo en cuenta los materiales y energía directos e indirectos (Infante-Amate, González de Molina, Toledo, 2017).

En este trabajo se utilizan dos indicadores específicos, centrándose en la producción desarrollada en América del Sur a fin de visibilizar los impactos ambientales del desarrollo.

\section{METOdOLOGÍA Y FUENTES}

\subsection{La huella ecológica}

El concepto huella ecológica fue introducido por Wackernagel y Rees a mediados de la década de los noventa, en relación con el concepto de capacidad de carga. Es una herramienta que permite analizar la demanda de tierra y espacio productivo por parte de la humanidad. Es un indicador biofísico de sostenibilidad que integra el conjunto de impactos que ejerce cierta comunidad humana sobre su entorno. Se define como "el área de tierra y agua biológicamente productiva que se necesita para producir los recursos que consume un individuo, población o actividad y para absorber los residuos que ello genera, considerando la tecnología y gestión de recursos imperante" (World Wildlife Fund, 2012: 135).

Para su dimensionamiento se le compara con el área productiva o biocapacidad disponible. Esta es entendida como la capacidad de los ecosistemas para producir materiales biológicos útiles y absorber los residuos humanos, utilizando los actuales esquemas de gestión y tecnologías de extracción. Establece de manera clara los límites del consumo de territorio productivo 5 .

La metodología de su cálculo se basa en la estimación de la superficie biológicamente productiva categorizada de la siguiente manera: tierras agrícolas, de pastoreo, pesquerías, áreas urbanizadas, bosques y áreas de absorción de

\footnotetext{
${ }^{5}$ La diferencia entre los valores de la huella ecológica y la biocapacidad permite conocer el nivel de deuda o de reserva ecológica existente en el ámbito de estudio. En caso de que un país o región posea una huella ecológica de la producción mayor a su biocapacidad-biocapacidad neta negativa- puede estar enfrentando dos situaciones: a) está colocando sus residuos en bienes comunes como pueden ser bosques y océanos, y consecuentemente deteriorando el capital natural mundial; y/o b) importando biocapacidad a través del comercio internacional de recursos naturales o de bienes con alto contenido de los mismos.
} 
dióxido de carbono. Cada categoría dispone de productividades biológicamente diferentes, por lo tanto, antes de sumarlas se procede a la normalización.

La huella ecológica tiene como una de sus principales ventajas su utilidad explicativa, dado que cuantifica de forma sencilla y comprensible, las demandas de recursos naturales -el binomio tierra/agua- necesarias para un determinado nivel o estilo de vida. Justamente, una de las críticas más fuertes se relaciona con que es un indicador quizás excesivamente sintético, con lo cual se encuentra demasiado agregado a nivel nacional e ignora diferencias en la productividad y en las formas de uso del suelo.

Debido a su forma de estimación, la huella ecológica tiende a infravalorar el peso de las actividades extractivas subterráneas al no tocar la biósfera y que no tienen un valor de rendimiento que pueda ser utilizado para traducir su creación en un área productiva, como las actividades petrolíferas, gasíferas y mineras. Estas limitaciones intentan ser sopesadas por la incorporación de los impactos de la energía y de otros materiales usados en la extracción, refinamiento, procesamiento y transporte de estos recursos minerales.

En este trabajo, tanto la huella ecológica como la biocapacidad se cuantifican en hectáreas globales (gha y los datos extraídos son del año 2007 y se encuentran disponibles en el informe World Wildlife Fund (2012).

\subsection{La huella hídrica}

Por su parte, el concepto de huella hídrica fue introducido por Hoekstra y Hung como un indicador para mapear el impacto del consumo humano de agua dulce y de su uso sostenible midiendo el volumen total utilizada directa o indirectamente por una población. Se originó en el intento de establecer nexos entre el consumo humano y el uso del agua, así como el comercio internacional con el manejo de los recursos hídricos.

Para el dimensionamiento de la huella hídrica se dice que el país es más autosuficiente en términos de recursos hídricos cuanto mayor sea la proporción de huella hídrica de productos locales sobre los importados. Por el contrario, si la proporción de huella hídrica de las importaciones es mayor, el país será más dependiente del agua de otras regiones del mundo. Sin embargo, ello no implica necesariamente dependencia en términos económicos o políticos.

La metodología de su cálculo se basa en la estimación de los cuatro componentes básicos que la conforman: el volumen, el color/clasificación, el lugar de origen, y el momento de extracción del agua. En cuanto al color, su clasificación 
se realiza a partir de la fuente originaria del agua: azul, verde o gris. Los usos alternativos, el manejo y los impactos para cada uno difieren significativamente:

TABLA 1. LOS COLORES DE LA HUELLA HÍDRICA

\begin{tabular}{|c|c|c|}
\hline Huella hídrica azul & Huella hídrica verde & Huella hídrica gris \\
\hline $\begin{array}{l}\text { Consumo de agua superfi- } \\
\text { cial -ríos, lagos, esteros- } \\
\text { y subterránea de determi- } \\
\text { nada cuenca, entendiendo } \\
\text { como consumo la extrac- } \\
\text { ción, con excepción del } \\
\text { agua utilizada que regresa } \\
\text { intacta al mismo lugar en un } \\
\text { período breve. }\end{array}$ & $\begin{array}{l}\text { Volumen de agua de lluvia eva- } \\
\text { porado o incorporado al pro- } \\
\text { ducto durante el proceso de pro- } \\
\text { ducción. } \\
\text { Particularmente relevante para } \\
\text { los productos agrícolas y fores- } \\
\text { tales, y refiere a la evapotrans- } \\
\text { piración del agua de lluvia total } \\
\text { de los campos y de las planta- } \\
\text { ciones, así como al agua incor- } \\
\text { porada a la cosecha o a la ma- } \\
\text { dera. } \\
\text { Nace a partir del agua verde } \\
\text { que consiste en el agua de lluvia } \\
\text { almacenada en el suelo como } \\
\text { humedad, siempre y cuando no } \\
\text { se convierta en lluvia escurrida } \\
\text { y extendida. }\end{array}$ & $\begin{array}{l}\text { Volumen de agua dulce que } \\
\text { se requiere para asimilar la } \\
\text { carga de contaminantes hasta } \\
\text { llegar a concentraciones que } \\
\text { cumplan con normas de cali- } \\
\text { dad de agua. } \\
\text { Es un indicador de la conta- } \\
\text { minación del agua dulce aso- } \\
\text { ciada a la fabricación de un } \\
\text { producto y su cadena de su- } \\
\text { ministro }\end{array}$ \\
\hline
\end{tabular}

Fuente: elaboración propia en base a revisión bibliográfica.

En este trabajo la huella hídrica se cuantifica en unidades de volumen de agua, generalmente en metros cúbicos). Los datos utilizados corresponden al período 1995-2006 disponibles en Mekonnen y Hoekstra (2011).

\section{EL DESARROLLO Y EL METABOLISMO SOCIOECONÓMICO DE AMÉRICA DEL SUR}

Los procesos de desarrollo económico de América del Sur y, por ende, su metabolismo socioeconómico, fueron variando a lo largo del tiempo. A finales del siglo XIX, América Latina se integró en el mercado mundial subordinada a la dinámica de los países industrializados mediante la provisión de materias primas. Sin embargo, luego de la crisis de los años treinta del siglo pasado, surgió un proceso de industrialización que determinó una rearticulación de buena parte de las economías latinoamericanas con la economía mundial. 
A finales del siglo XX y comienzos del siguiente, las ideas surgidas de las corrientes que problematizaban el desarrollo con base industrial fueron silenciadas por teorías que sostenían que el libre comercio, la libre movilidad del capital financiero, el desarrollo sostenible y la fuerte expansión de las empresas trasnacionales que, supuestamente, iban a generar nuevas oportunidades de desarrollo para los países atrasados o en vías de desarrollo. El correlato económico de estas nuevas políticas de desarrollo derivó en una alta concentración de los mercados, la hegemonía del capital trasnacional y una creciente dependencia de la extracción/producción de recursos naturales para la exportación (Belloni, Wainer, 2014).

Los resultados de estos procesos históricos son los que se pueden reflejar en una nueva estructura socioeconómica que a su vez se puede visibilizar con los indicadores biofísicos antes mencionados.

\subsection{La huella ecológica de América del Sur}

En el año 2007 la huella ecológica total de la humanidad alcanzaba los 18.200 millones de hectáreas globales, que definimos por el acrónimo gha; esto es, 2,7 gha/persona), Como contraste, la biocapacidad total de la Tierra era de 12.000 millones de gha, lo que equivale a 1,8 gha/persona. El planeta presentó así un déficit ecológico de 6.200 millones de gha o 0,92 gha/persona) ${ }^{6}$ (World Wildlife Fund, 2012).

Sin embargo, la huella ecológica de América Latina posee no sólo una huella per cápita inferior a la mundial, sino que además se presenta como una importante fuente de biocapacidad para la humanidad, registrando valores per cápita superiores al promedio mundial. Brasil posee una biocapacidad que representa el 15,37\% del total del planeta - encontrándose así entre los diez países con mayores niveles del mundo-, al tiempo que Argentina representa el 2,36\% de la biocapacidad mundial.

El estudio de la huella ecológica en términos desagregados se centra en buscar los principales sectores y actividades determinantes de la misma. Así, se muestra que mientras a nivel mundial la principal huella ecológica son las de emisiones de $\mathrm{CO}_{2}$, mayoritariamente asociadas a las actividades industriales, transporte y a determinados bienes de consumo relativamente masivo. Sin embargo, para América del Sur el principal determinante es la huella ecológica agro-

\footnotetext{
${ }^{6}$ Estos datos significan que la Tierra tardaría en 2007 1,5 años en regenerar completamente los recursos renovables que los seres humanos utilizan en 1 año.
} 
pecuaria, vinculado a las tierras de cultivo y de pastoreo, y al mencionado sector exportador (Belloni, Peinado, 2013).

\footnotetext{
GrÁFICO 1. Mundo y AmÉRICA DEL Sur. DisTribuCión DE LA HUElla ECOLÓGICA DE LA PRODUCCIÓN POR GRANDES CATEGORÍAS, 2007 (EN GHA POR PERSONA)
}

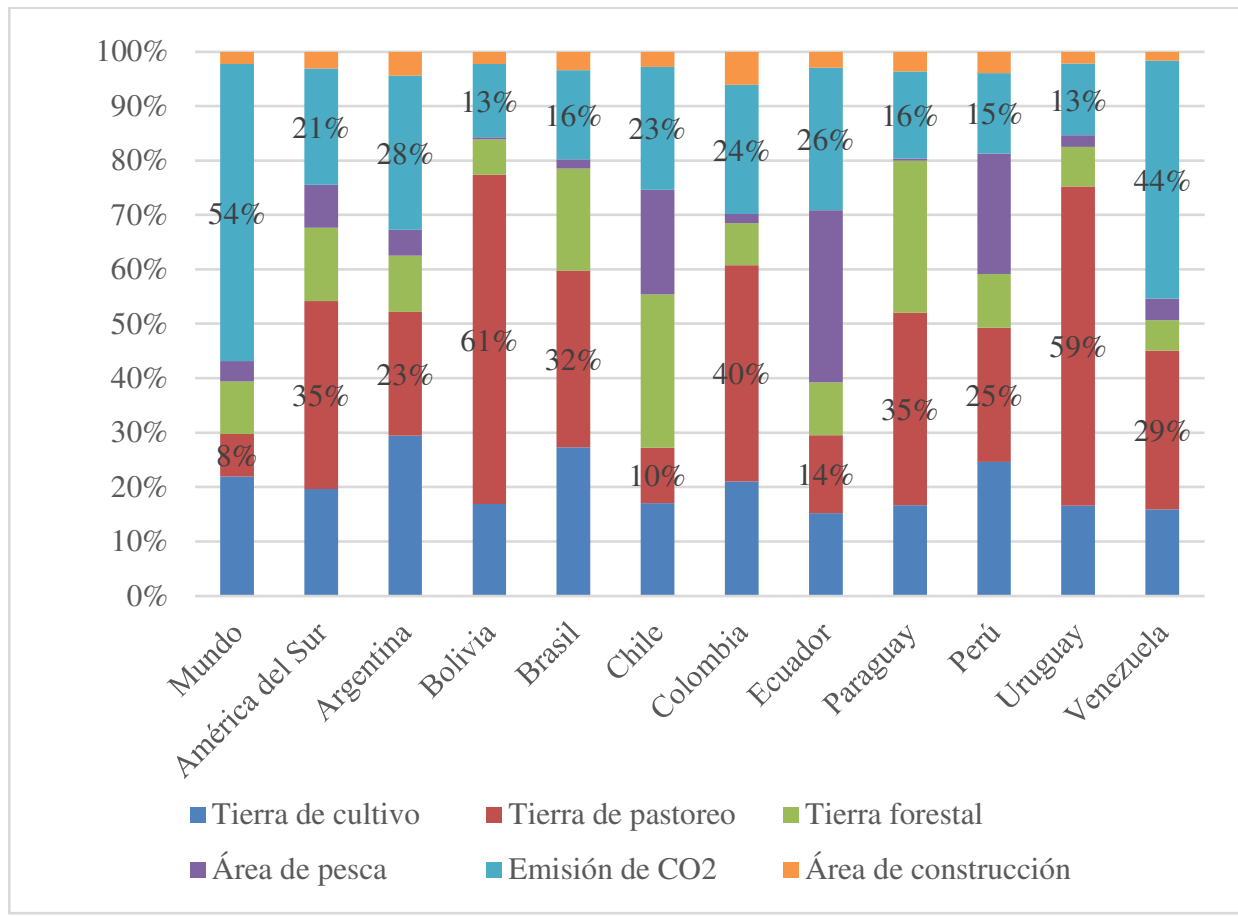

Fuente: elaboración propia en base a datos de Global Footprint Network en World Wildlife Fund (2012).

En el Gráfico 1 se puede visualizar que mientras a nivel mundial la huella de carbono ${ }^{7}$ representaba el 54\% de la huella ecológica, en América del Sur representa un $21 \%$. Con excepción de Venezuela, para el resto de los países de América del Sur el principal determinante de la huella ecológica es la referida al sector agropecuario; esto es, tierras de cultivo y de pastoreo. Tanto es así que la huella ecológica del sector agropecuario en América del Sur representa el 53\% de la huella ecológica, mientras a nivel mundial representa el 30\%. Entre los principales países con una elevada huella ecológica generada por el sector agropecuario encontramos a Bolivia, con un 78\%; Uruguay, con un 76\%; Colombia, con

\footnotetext{
${ }^{7}$ La huella de carbono mide la cantidad de capacidad biológica, en hectáreas globales, exigida por las emisiones humanas de dióxido de carbono fósil y refiere a la cantidad de gases de efecto invernadero emitidos durante el ciclo de vida de un producto a lo largo de la cadena de producción.
} 
un 61\%-; Brasil, con un 59\%; y Paraguay, con un 53\%. Esto ocurre debido a la inserción de América Latina en el comercio internacional y a que su industrialización está acotada a la estructura exportadora intensiva de recursos naturales.

Paralelamente, a partir del Gráfico 2 y del Gráfico 3 se aprecia que, en términos generales, los países de América del Sur poseen huellas ecológicas de la producción por debajo de la biocapacidad con que cuentan. Esto significa que dichos países tienen un superávit ecológico ya que la capacidad disponible capacidad que tienen sus ecosistemas de abastecer recursos naturales útiles y absorber los residuos es mayor que la presión ambiental que ejercen estas economías. En este escenario se distinguen Bolivia, Brasil, Paraguay, Colombia y Perú que, simultáneamente, poseen una elevada biocapacidad per cápita y bajas huellas ecológicas per cápita de la producción. En el otro extremo, hay países que ejercen una presión ambiental mayor que la capacidad de sus ecosistemas. Un ejemplo es Chile, que tiene una huella ecológica per cápita que excede su biocapacidad per cápita.

GRÁfiCo 2. AMÉRICA DEL SuR. HuELla ECOLÓGICA Y BIOCAPACIDAD, 2007 (EN GHA POR PERSONA)

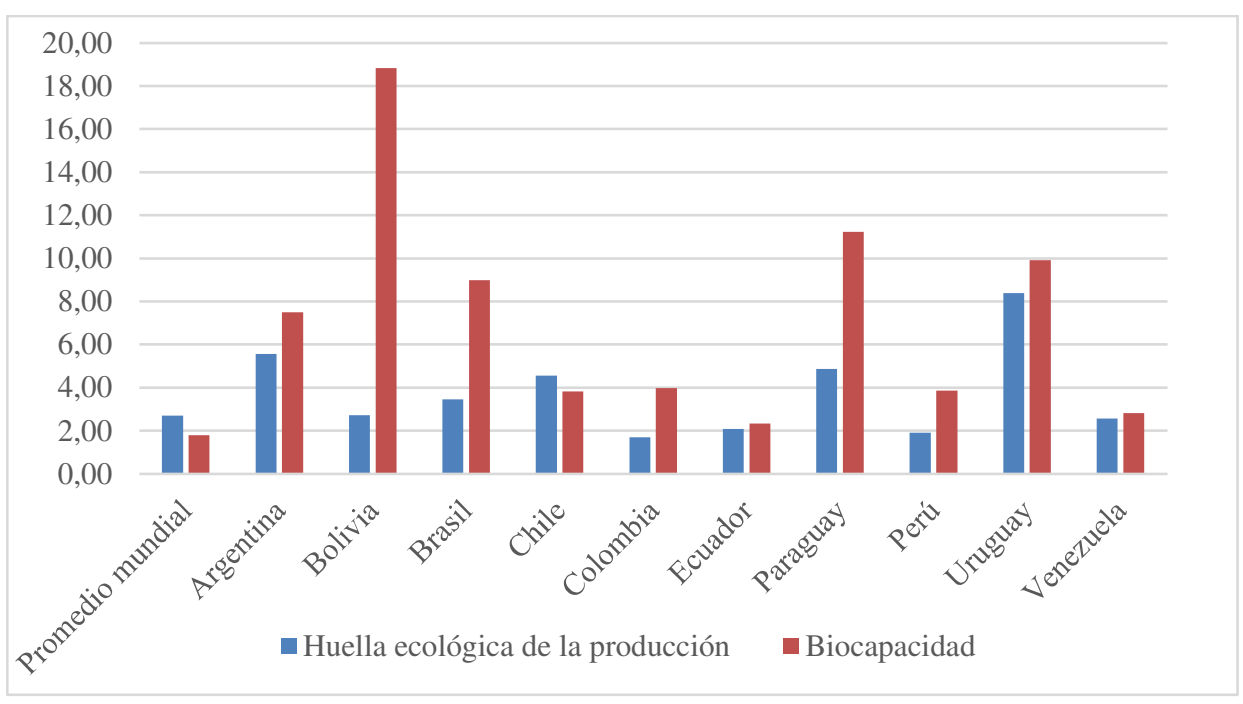

Fuente: elaboración propia en base a datos de Global Footprint Network en World Wildlife Fund (2012).

Aquellos países que tienen un comportamiento similar al de Chile, y el agregado Mundo, - es decir que poseen una huella ecológica de la producción mayor a su biocapacidad, o sea, biocapacidad neta negativa-, pueden estar enfrentando dos situaciones: a) están colocando sus residuos en bienes comunes y consecuentemente deteriorando su propio capital natural; y/o b) importan bio- 
capacidad a través del comercio internacional de recursos naturales o de bienes con alto contenido de los mismos, lo que podría derivar en un intercambio ecológicamente desigual con otro país ${ }^{8}$.

GrÁFICO 3. AmÉriCA DEL Sur. HuElla ECOLÓGICA COMO PORCENTAJE DE LA BIOCAPACIDAD, 2007 (EN PORCENTAJES)

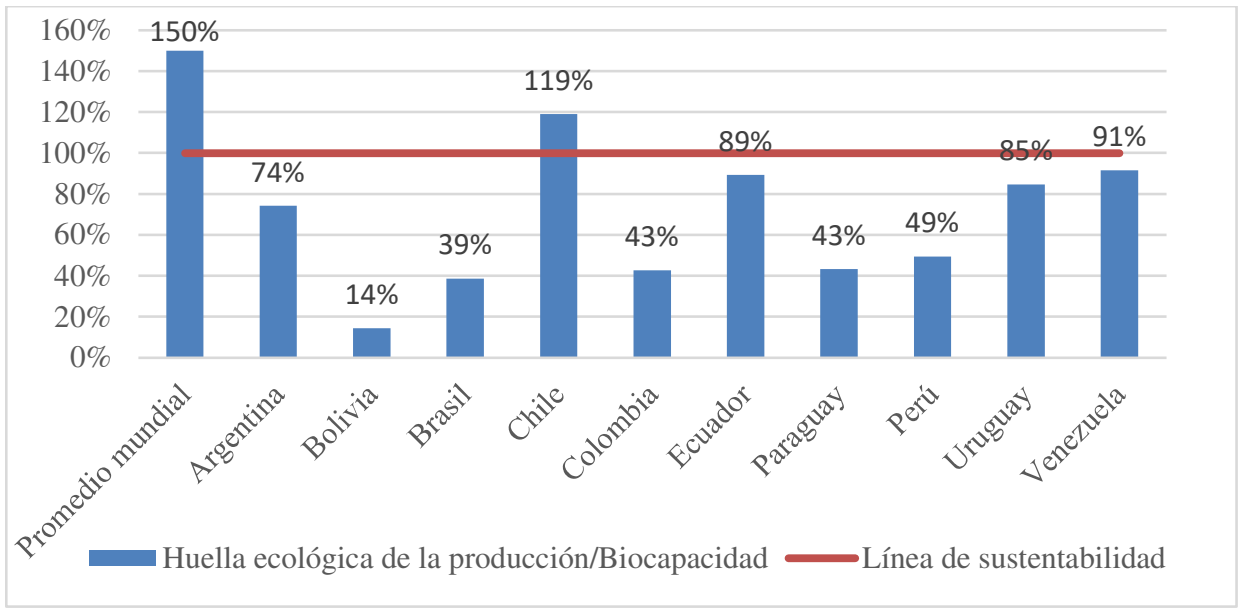

Fuente: elaboración propia en base a datos de Global Footprint Network en World Wildlife Fund (2012).

Sin embargo, cabe aclarar que el sistema mundo es un sistema cerrado que no puede obtener biocapacidad de otros sistemas. Por lo tanto, como demuestran el Gráfico 2 y el Gráfico 3, el planeta Tierra posee una huella ecológica mayor que su biocapacidad. Ello implica que se están colocando los residuos en bienes comunes y, consecuentemente, deteriorando el capital natural a nivel mundial.

\subsection{La huella hídrica de América del Sur}

Otra de las formas de cuantificar el impacto de las actividades humanas sobre el ambiente es a través de la huella hídrica, la cual pone su énfasis en la cuantificación de los usos del agua como recurso central para la reproducción de las vidas.

La huella hídrica depende de los distintos patrones de consumo doméstico y de la inserción internacional que posee cada país. A nivel mundial, para el periodo 1995-2006, la huella hídrica de una persona promedio fue de 1.169 Mm³/año -azul

\footnotetext{
${ }^{8}$ Sobre el concepto de intercambio ecológicamente desigual ver Hornborg y Martínez Alier (2016), Jorgenson (2016) y Peinado (2015). En relación al intercambio ecológicamente desigual en Latinoamérica ver West y Schandl (2013) y en referencia en América del Sur ver Belloni y Peinado (2013).
} 
más verde-, representando los productos agrícolas el 92\% de esta huella; el 5\%, los productos industriales; y el 4\%, el uso doméstico ${ }^{9}$ (Mekonnen, Hoekstra, 2011).

En cuanto a los flujos de recursos hídricos implícitos en el comercio internacional, se distinguen entre los mayores exportadores Estados Unidos, Brasil, India, Argentina, Australia, Canadá y China. Entre los mayores importadores se encuentran Estados Unidos, China, Japón, Alemania, Italia y México (Mekonnen, Hoekstra, 2011). De esta manera, tanto Argentina como Brasil son grandes proveedores mundiales de recursos hídricos a través del comercio internacional de bienes.

América del Sur representa el 5,66\% de la población mundial, implica el $3,85 \%$ del PIB mundial y tiene una participación similar tanto en exportaciones como en importaciones en términos monetarios, con una tendencia a presentar mayores exportaciones que importaciones; esto es, proveedor neto de bienes para el resto del mundo.

Ahora bien, cuando se tiene en cuenta la intensidad en agua de las exportaciones e importaciones, se encuentra que mientras el 2,53\% de la participación en las importaciones en dólares crece apenas al 3,49\% en concepto de huella hídrica, las exportaciones en dólares que representaban un 3,07\% crecen fuertemente a un $11,35 \%$ a nivel mundial.

TABlA 2. AMÉRICA DEL SuR. PRINCIPALES DATOS AGREGADOS. 1996-2005. (EN PORCENTAJES DE POBLACIÓN, MM $M^{3}$ Y DÓLARES CORRIENTES)

\begin{tabular}{|l|c|}
\hline Categoría & América del Sur \\
\hline Participación en la población mundial & $5,66 \%$ \\
\hline Participación en el PIB mundial & $3,85 \%$ \\
\hline Participación en las exportaciones mundiales en dólares EE.UU. & $3,07 \%$ \\
\hline Participación en las exportaciones mundiales de huella hídrica & $11,35 \%$ \\
\hline Participación en las importaciones mundiales en dólares EE.UU. & $2,53 \%$ \\
\hline Participación en las importaciones mundiales de huella hídrica & $3,49 \%$ \\
\hline
\end{tabular}

Fuente: elaboración propia en base a datos de Banco Mundial, COMTRADE y Mekonnen y Hoeks$\operatorname{tra}(2011)$.

\footnotetext{
${ }^{9}$ Los países centrales presentan huellas hídricas per cápita entre 1.008 y $2.207 \mathrm{Mm} 3$ /año, mayores que el promedio.
} 
A partir de ello, se empieza a visibilizar que el perfil de las exportaciones de América del Sur es muy fuerte en recursos hídricos -no tanto sus importaciones- y que estos países, además de ser proveedores netos de bienes, son importantísimos proveedores netos de recursos hídricos implícitos en sus exportaciones ${ }^{10}$. Esto vuelve a reforzar la idea de un patrón de exportaciones basado en el sector primario y de un intercambio ecológicamente desigual (Belloni, Peinado, 2013; Peinado, 2018).

GRÁFICO 4. AMÉRICA DEL SUR. DistRIBUCIÓN DE LA HUELLA HÍDRICA DE LA PRODUCCIÓN. 1996-2005 $\left(E N M M^{3}\right)$

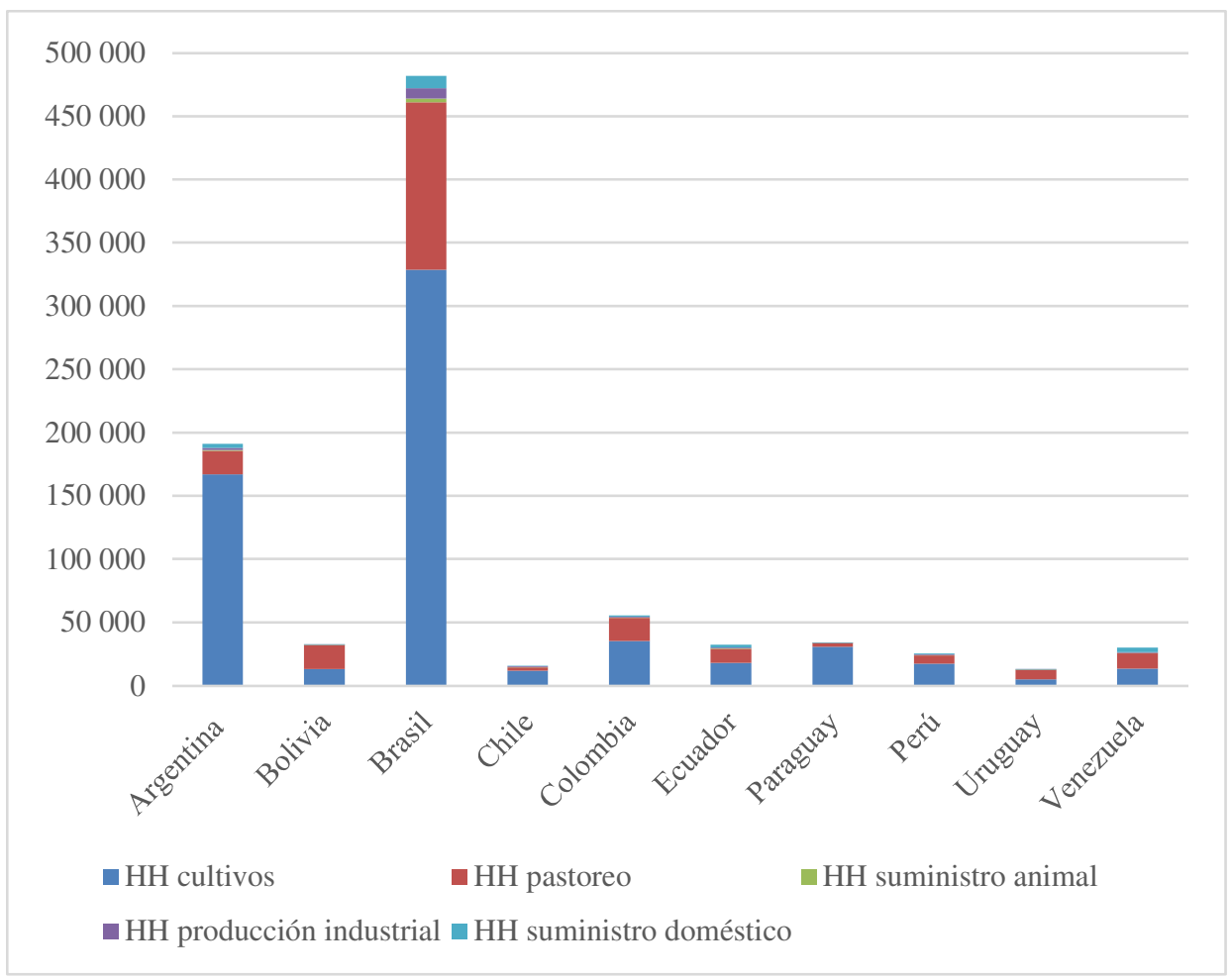

Fuente: elaboración propia en base a datos de Mekonnen y Hoekstra (2011).

Si bien -como se dijo anteriormente y como explicita en el Gráfico 4Brasil y Argentina son preponderantes en términos de exportaciones de huella hídrica, esa relevancia se reduce parcialmente al trabajar los valores en términos

\footnotetext{
${ }^{10}$ Concretamente, Brasil y Argentina ocupan el cuarto y quinto lugar respectivamente entre los mayores exportadores mundiales de huella hídrica (Mekonnen, Hoekstra, 2011).
} 
per cápita. Allí, la preponderancia de Brasil es drásticamente reducida; no así en el caso de Argentina, que luego de Paraguay, tiene la segunda mayor huella hídrica per cápita de América del Sur, siendo esta muy superior a la mundial, tal y como se aprecia en el Gráfico 5.

GRÁFICO 5. AMÉRICA DEL SUR. DiSTRIBUCIÓN DE LA HUELLA HÍDRICA DE LA PRODUCCIÓN. 1996-2005 (EN MM ${ }^{3}$ PER CÁPITA)

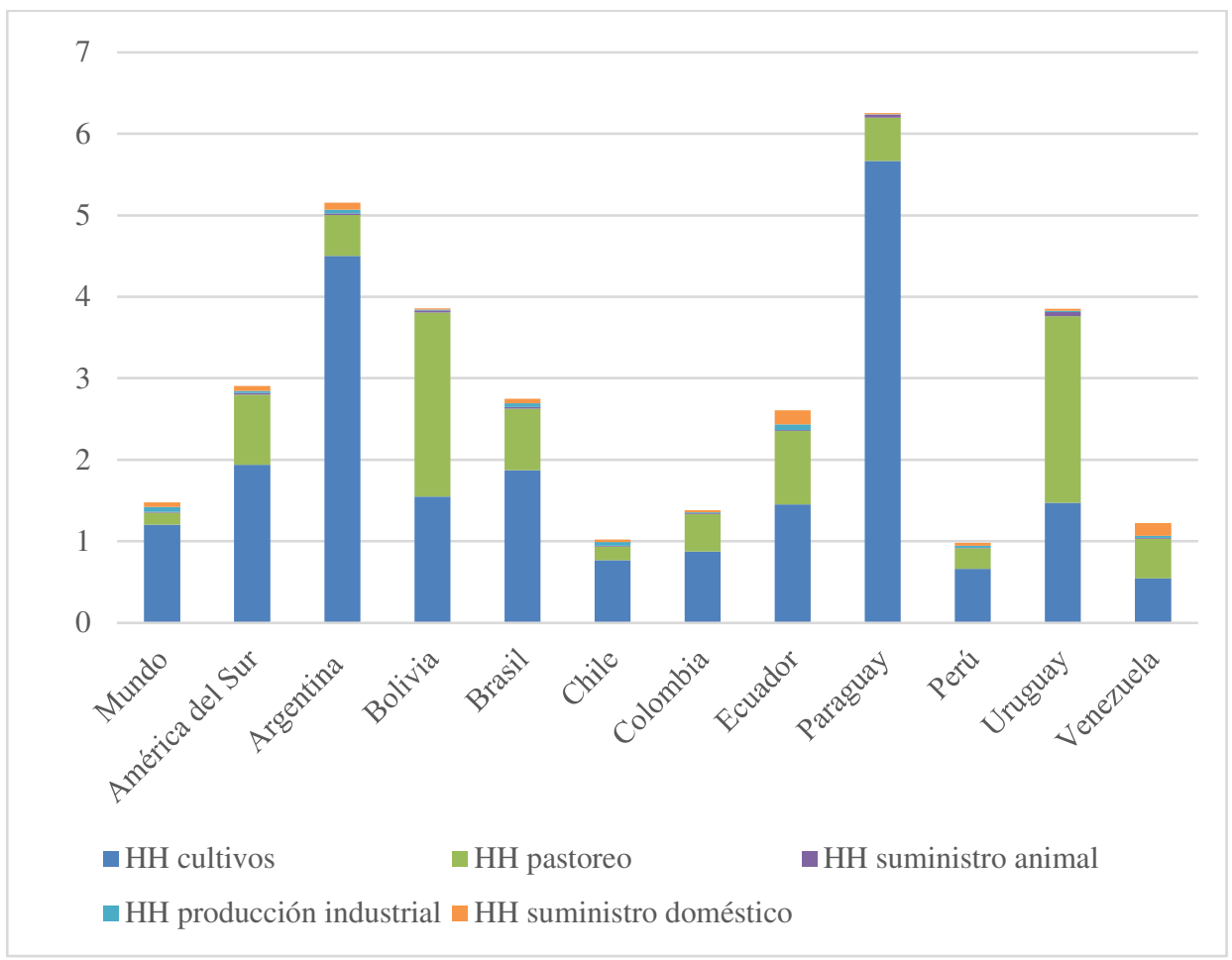

Fuente: elaboración propia en base a datos de Mekonnen y Hoekstra (2011).

En cuanto a la estructura interna de esas huellas hídricas -véase el Gráfico 6- se destaca, primeramente, cómo a nivel mundial tienen una mayor significancia las huellas asociadas a la industria y al suministro doméstico. Ello cual también es reflejo de una estructura productiva de América del Sur que no está orientada ni a la generación de empleo, ni a la generación de condiciones de vida dignas para su población ${ }^{11}$. Por otro lado, se aprecia la preponderancia de la huella hídrica de cultivos, con la excepción de Bolivia y Uruguay.

${ }^{11}$ Con la excepción de Venezuela, Ecuador y Chile. 
GRÁFICO 6. Mundo Y AMÉRICA DEL SUR. COMPOSICIÓN DE LA HUELLA HÍDRICA DE LA PRODUCCIÓN. 1996-2005 (EN PORCENTAJE)

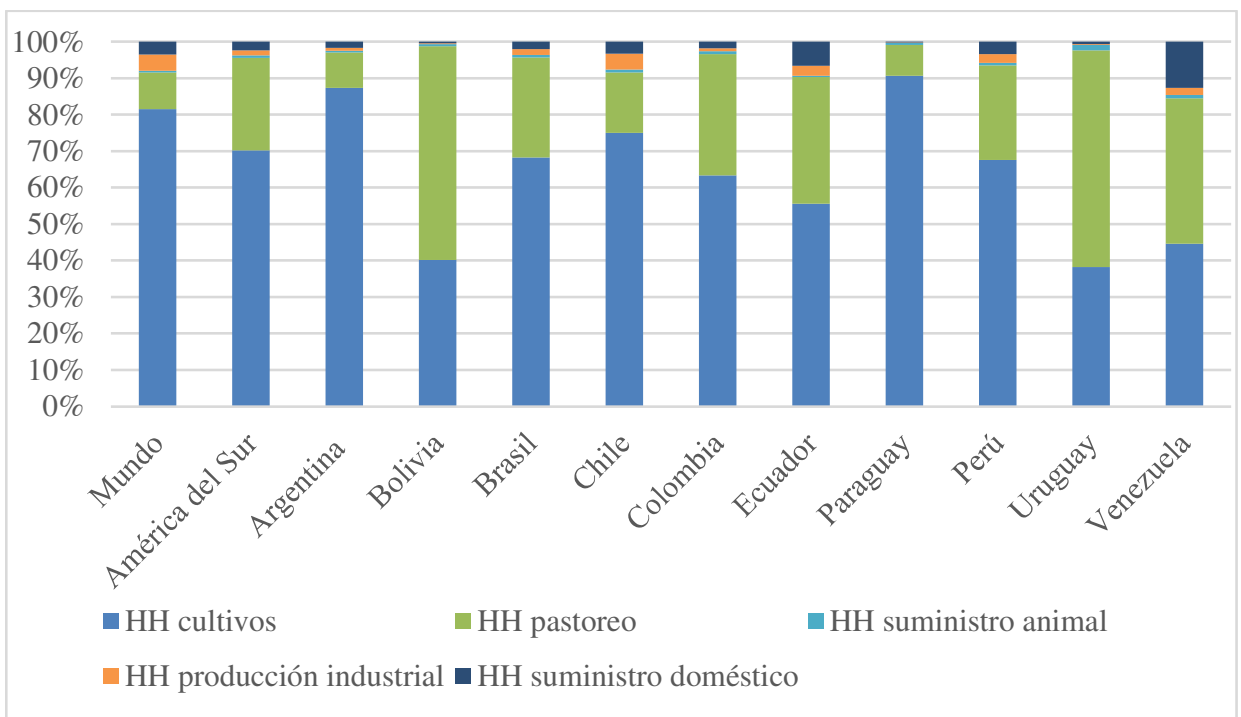

Fuente: elaboración propia en base a datos de Mekonnen y Hoekstra (2011).

En relación con las condiciones de vida de la población, en el Gráfico 7 se destaca la huella per cápita del suministro doméstico de Ecuador y Venezuela, países en los que hay un peso significativo en términos absolutos -aunque no así en términos relativos- de la huella azul.

GrÁFICO 7. Mundo y AMÉRICA DEL SuR. COMPOSICIÓN DE LA HUELLA HÍDRICA DE SUMINISTRO DOMÉSTICO. 1996-2005 (EN MM³ PER CÁPITA)

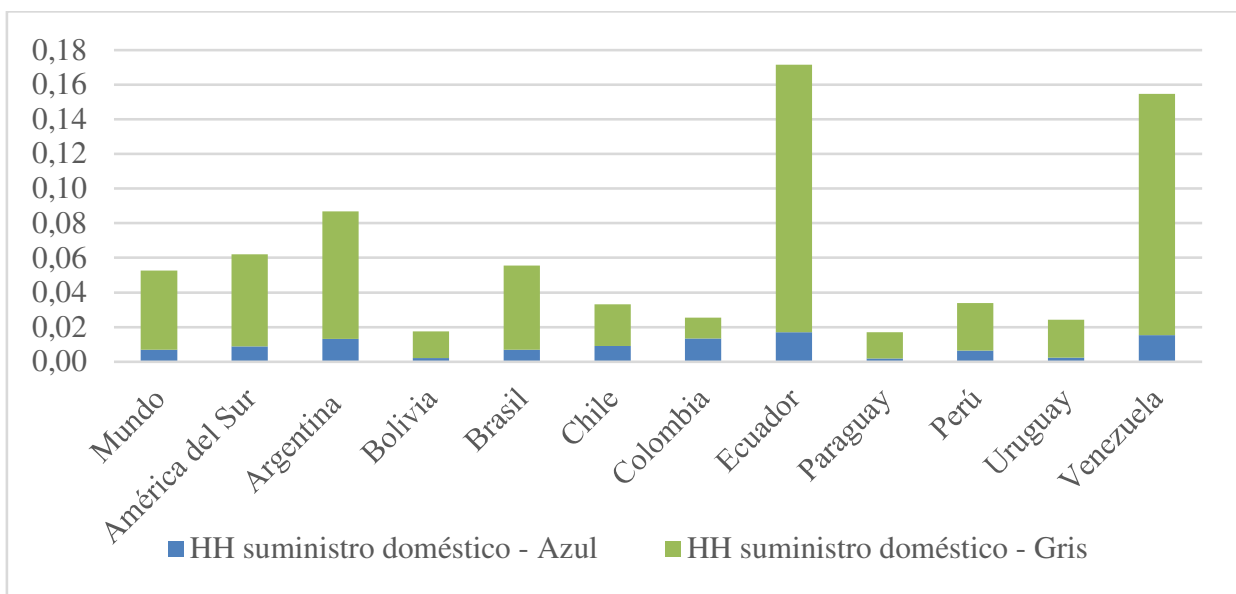

Fuente: elaboración propia en base a datos de Mekonnen y Hoekstra (2011). 
En lo que refiere a la intensidad hídrica del sector industrial a la hora de incorporar valor -véase el Gráfico 8-, se evidencia que mientras el promedio mundial es de 42,95 $\mathrm{m}^{3}$ por millar de dólares EE.UU., el promedio en América del Sur es de 34,74, lo cual indica que la industria en América del Sur es menos intensiva en recursos hídricos que el promedio mundial. Esto evidencia que el perfil industrial de América del Sur no está centrado en actividades hídricas intensivas, aunque con claras excepciones como Ecuador. La contrapartida negativa es el elevado porcentaje de huella hídrica gris, con las excepciones de Chile y Perú.

GRÁFICO 8. MUNDO Y AMÉRICA DEL SUR. INTENSIDAD HÍDRICA DEL SECTOR INDUSTRIAL. HUELLA HÍDRICA PROMEDIO POR UNIDAD MONETARIA DE VALOR INDUSTRIAL AGREGADO. 1996-2005 (EN $\left.M^{3} / M I L L A R U S D\right)$

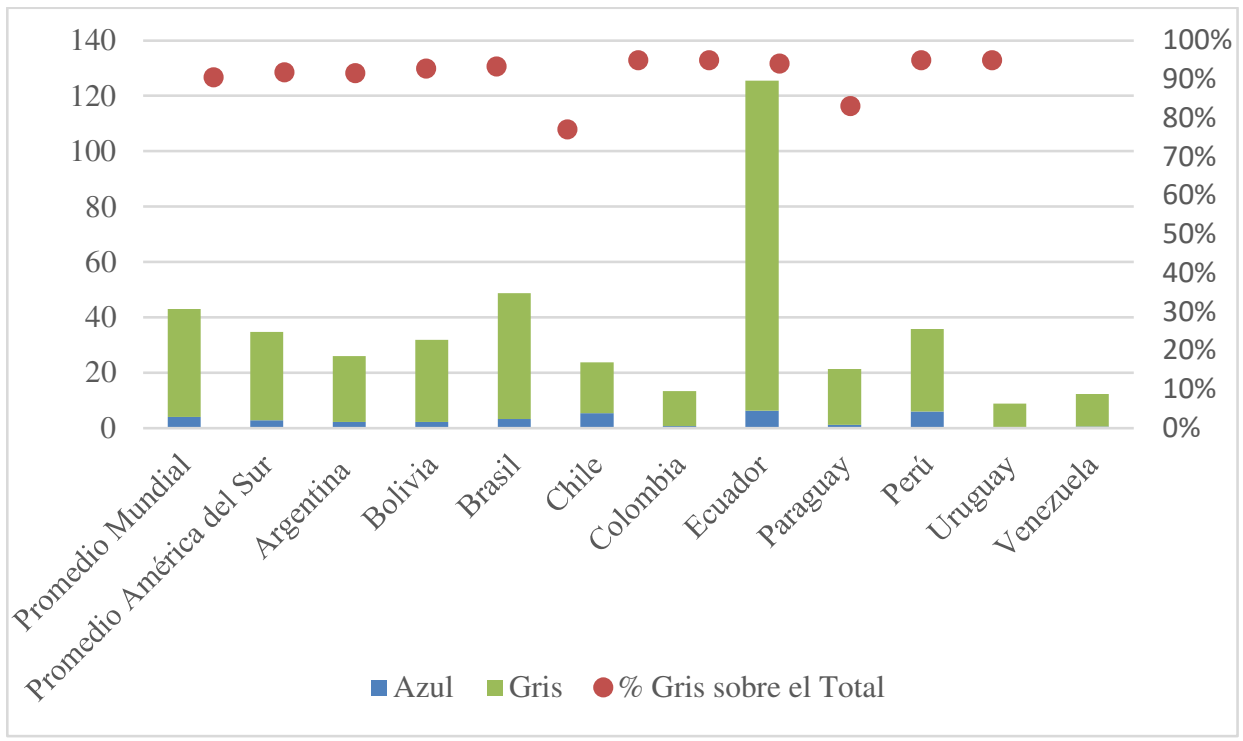

Fuente: elaboración propia en base a datos de Mekonnen y Hoekstra (2011).

En este sentido, se destaca la mayor ineficiencia respecto a la media mundial de la industria en Ecuador, principalmente, y de Brasil. En el otro extremo, se destacan Uruguay, Venezuela y Colombia, con un elevado valor monetario en relación a los recursos hídricos.

En cuanto al peso de la huella hídrica gris sobre el total, se destacan Chile y Perú con industrias que, si bien no se destacan como generadoras de valor monetario en relación a los recursos hídricos utilizados, sí lo hacen con una menor presencia de la huella gris. 


\section{CONCLUSIONES}

Las últimas décadas del siglo XX y el comienzo del siglo XXI han colocado en el centro del debate económico la cuestión ambiental. En un principio, y a través de los objetivos del desarrollo sostenible, se logró disociar los problemas ambientales del ámbito de la producción. El artículo 10 del Acuerdo de París ${ }^{12}$ afirma: "para dar una respuesta eficaz y a largo plazo al cambio climático y promover el crecimiento económico y el desarrollo sostenible es indispensable posibilitar, alentar y acelerar la innovación". En este acuerdo se vuelve a retomar la idea de desarrollo sostenible que no cuestiona la idea del crecimiento ilimitado, cuando ya son evidentes sus consecuencias socioambientales a nivel global. Tampoco se reconoce ni el intercambio ecológicamente desigual entre países centrales y periféricos ni la deuda ecológica (Rice, 2009; Acosta, Brand, 2017; Peinado, 2018).

La idea de un desarrollo sostenible -o de desarrollos alternativos sin cuestionar que el crecimiento económico- implica mayor extracción de materiales y energía. Ello revela la actitud gerencial del planeta Tierra, en donde lo que está en juego es la continuidad de los modelos de crecimiento y desarrollo a través de estrategias administrativas adecuadas (Escobar, 2014). Este nuevo discurso desarrollista intenta lograr una política de consenso disolviendo cualquier contradicción que pudiese haber entre sostenibilidad económica y sustentabilidad ambiental.

En este trabajo se ha centrado en visibilizar a través de indicadores biofísicos, primeramente, las inequidades en los metabolismos socioeconómicos entre América del Sur y el promedio mundial. En ambas huellas el resultado es claro: América del Sur tiene un perfil productivo y un patrón de comercio que es producto de una estructura económica que basa sus exportaciones en materias primas o derivados de éstas. La idea del desarrollo sustentable aplicable a nuestra región se tradujo en un incremento de este intercambio ecológicamente desigual y una reducción del capital natural de América del Sur.

En el caso de la huella hídrica, al observar la intensidad en agua de las exportaciones e importaciones en América del Sur, se encuentra que mientras el 2,53\% de la participación en las importaciones en dólares crece apenas al 3,49\% de las importaciones de huella hídrica, las exportaciones en dólares que representaban un $3,07 \%$ crecen fuertemente a un $11,35 \%$ a nivel mundial.

\footnotetext{
12 Acuerdo dentro del marco de la Convención Marco de las Naciones Unidas sobre el Cambio Climático que establece medidas para la reducción de las emisiones de Gases de Efecto Invernadero (GEI) aplicable para el año 2020, cuando finaliza la vigencia del Protocolo de Kioto. El acuerdo fue negociado por los 195 países miembros, adoptado el 12 de diciembre de 2015 y abierto para firma el 22 de abril de 2016.
} 
Si se toma el indicador de huella ecológica, se aprecia exactamente lo mismo: mientras el promedio mundial consume un $50 \%$ más de su biocapacidad, en América del Sur todos los países, con excepción de Chile, tienen su huella ecológica por debajo de la biocapacidad. Esto significa que el déficit ecológico mundial sería muchísimo más profundo si en Sudamérica no tuviese dicho superávit.

Durante este último siglo volvieron a ponerse en marcha políticas desarrollistas en varios países de la región. Sin embargo, ninguno de estos países pudo cambiar el metabolismo socioeconómico característico de América del Sur. Las ideas desarrollistas no sólo no consiguieron resolver el dilema de una estructura exportadora intensiva en recursos naturales, sino que la profundizaron (West \& Schandl, 2013).

Esta disyuntiva, histórica pero también actual, merece ser abordada mediante nuevos esquemas que permitan pensar alternativas a la idea de desarrollo. Las nuevas políticas de decrecimiento (D'Alisa, Demaria, Kallis, 2017) y la idea de posextractivismo (Acosta, Brand, 2017), el buen vivir o vivir bien de los países andinos (Endara, 2014; Cubillo-Guevara, Hidalgo-Capitán, García-Álvarez, 2016) y las prácticas económicas locales (Mora, 2018) merecen ser abordadas con el objetivo de reducir las desigualdades sociales y ambientales y enfrentar la crisis socioambiental en la que vivimos.

\section{BIBLIOGRAFÍA}

Acosta, A., Brand, U. (2017). Salidas del laberinto capitalista. Decrecimiento y Posextractivismo. Buenos Aires, Argentina: Tinta Limón, Fundación Rosa Luxemburgo.

Belloni, P., Peinado, G. (2013). Inserción externa, capitales transnacionales e intercambio ecológicamente desigual en la América del Sur posneoliberal. Sociedad y Economía, (25), pp. 15-38.

Belloni, P., Wainer, A. (2014). El rol del capital extranjero y su inserción en la América del Sur posneoliberal. Problemas del desarrollo, 45(177), pp. 87-115. https://doi.org/10.1016/S0301-7036(14)70864-8

Cubillo-Guevara, A., Hidalgo-Capitán, A., García-Álvarez, S. (2016). El Buen Vivir como alternativa al desarrollo para América Latina. Iberoamerican Journal of Development Studies, 5(2), pp. 30-57. https://doi.org/10.26754/ojs_ried/ijds.184

D'Alisa, G., Demaria, F., Kallis, G. (2017). Decrecimiento. Vocabulario para una nueva era. Buenos Aires, Argentina: Biblioteca Permacultura-Icaria.

Daly, H. (2013). Una economía de estado estacionario. Nueva Sociedad, (244), pp. 134-141.

Endara, G. (2014). Post-crecimiento y buen vivir. Quito, Ecuador: Friedrich Ebert Stiftung.

Escobar, A. (2014). Sentipensar con la tierra. Nuevas lecturas sobre desarrollo, territorio y diferencia. Medellín, Colombia: Universidad Autónoma Latinoamericana (UNAULA). 
Estenssoro Saavedra, F., Vásquez Bustamante, J. P. (2018). Perspectivas políticas ambientales latinoamericanas. Estudios Hemisféricos y Polares, 9(3), pp. 1-26.

Fischer-Kowalski, M. (1997). Society's metabolism: on the childhood and adolescence of a rising conceptual star. En M. Redclift, G. Woodgate (eds.), The International Handbook of Environmental Sociology (pp. 119-137). Cheltenham, Reino Unido: Edward Elgar. https://doi.org/10.1162/jiec.1998.2.1.61

Fischer-Kowalski, M. (1998). Society's Metabolism. The Intellectual History of Materials Flow Analysis, Part I, 1860-1970. Journal of Industrial Ecology, pp. 2(1), 61-78.

Hornborg, A., Martínez Alier, J. (2016). Ecologically unequal exchange and ecological debt. Journal of Political Ecology, 23(1), pp. 328-491. https://doi.org/10.2458/v23i1.20220

Infante-Amate, J., González de Molina, M., Toledo, V. (2017). El metabolismo social. Historia, métodos y principales aportaciones. Revista Iberoamericana de Economía Ecológica, (27), pp. 130-152.

Jorgenson, A. (2016). The sociology of ecologically unequal exchange, foreign investment dependence and environmental load displacement: summary of the literature and implications for sustainability. Journal of Political Ecology, 23(1), pp. 334-349. https://doi.org/10.2458/v23i1.20221

Martínez Alier, J., Sejenovich, H., Baud, M. (2015). El ambientalismo y ecologismo latinoamericano. En F. D. Castro, M. Baud (eds.), Gobernanza ambiental en América Latina (pp. 3973). Buenos Aires, Argentina: CLACSO.

Mekonnen, M. M., \& Hoekstra, A. Y. (2011). National Water Footprint Accounts: The Green, Blue and Grey Water Footprint of Production and Consumption. Value of Water. Research Report Series (50).

Mora, A. (2018). Prácticas Económicas de Base. Buenos Aires, Argentina: Friedrich Ebert Stiftung.

Organizaciones de las Naciones Unidas (ONU). (1987). Nuestro Futuro Común. Nairobi: ONU.

Peinado, G. (2015). Intercambio ecológicamente desigual e Intercambio desigual en Oscar Braun Nexos, puntos en común y especificidades. Revista Iberoamericana de Economía Ecológica (REVIBEC), (24), pp. 187-202.

Peinado, G. (2018). Economía Ecológica y comercio internacional: el intercambio ecológicamente desigual como visibilizador de los flujos ocultos del comercio internacional. Revista Economía, 70(112), pp. 53-69. https://doi.org/10.29166/economia.v70i112.2046

Rice, J. (2009). North-South Relations and the Ecological Debt: Asserting a Counter-Hegemonic Discourse. Critical Sociology, 35(2), pp. 225-252. https://doi.org/10.1177/0896920508099193

Toledo, V. (2013). El metabolismo social: una nueva teoría socioecológica. Relaciones, (136), pp. 41-71.

West, J., Schandl, H. (2013). Tendencias del flujo de materiales y productividad de recursos en América Latina. Ciudad de Panamá, Panamá: Programa de las Naciones Unidas para el Medio Ambiente.

World Wildlife Fund. (2012). Living Planet Report 2012. Biodiversity, biocapacity and better choices. Gland, Suiza: WWF International. 